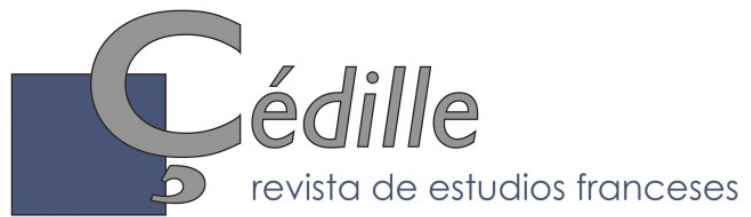

ISSN: $1699-4949$

no 19 (primavera de 2021)

Monografías 12

Linguistique textuelle et graphématique du français en diachronie

Elena Llamas-Pombo \& Diana Gatea, editoras científicas

\title{
La formation de la textualité en diachronie : jonctions interpropositionnelles entre le français médiéval et le français moderne
}

\author{
Soili HAKULINEN \\ Tampere University \\ soili.hakulinen@tuni.fi \\ https://orcid.org/0000-0003-0632-2193
}

\section{Resumen}

Este artículo examina la evolución de la textualidad en francés entre los siglos XIV y $\mathrm{XX}$, a través de las conexiones entre proposiciones, presentes en un corpus formado por dos traducciones de un mismo texto (Boecio, Consolatio Philosophiae) a través de diferentes épocas. Nuestra hipótesis, confirmada por nuestro análisis, es que la textualidad del francés del siglo XIV (etapa «joven» en que la grafía aún no está fijada) es más agregativa y concentrada que la textualidad del francés moderno, influida por una larga tradición literaria anterior.

Palabras clave: textualidad, conexión entre proposiciones, agregación, integración, puntuación.

\section{Résumé}

Le présent article s'intéresse à l'évolution de la textualité en français entre le $\mathrm{XIV}^{\mathrm{e}}$ et le $\mathrm{XX}^{\mathrm{e}}$ siècle à travers les jonctions interpropositionnelles. La textualité est examinée sur un corpus parallèle de deux traductions d'un même texte (Boèce, Consolatio Philosophiae) aux époques concernées. Selon l'hypothèse, qui se trouve confirmée par l'analyse du corpus, la textualité du français au XIV $\mathrm{XV}^{\mathrm{e}}$ siècle, époque où la langue écrite est jeune et les conventions de l'écriture ne sont pas encore fixes, est plus agrégative, moins serrée, que celle de l'époque moderne où la langue a une longue tradition littéraire derrière elle.

Mots-clés : textualité, jonctions interpropositionnelles, agrégation, intégration, ponctuation.

\begin{abstract}
:
This article explores the evolution of textuality in French between the fourth and the twentieth century by studying interpropositional junctions. Textuality is analyzed in a parallel corpus of two translations of the same text (Boethius, Consolatio Philosophiae) from these two periods. The hypothesis, which is confirmed by the analysis of the corpus, is that the textuality
\end{abstract}

*Artículo recibido el 15/09/2020, aceptado el 30/03/2021. 
of French in the fourth century - a period in which the written language was young and its conventions were not yet fixed - was more aggregative and less condense than in the modern period, when French has a long tradition of writing behind it.

Key words: textuality, interpropositional junctions, aggregation, integration, punctuation.

\section{Introduction}

Comme toute langue, la langue écrite évolue avec le temps. Elle peut acquérir des caractéristiques textuelles plus complexes et plus serrées, comme cela a été montré pour l'espagnol du Moyen Âge lors des débuts de cette langue écrite, ou pour la presse rhéto-romane en Suisse à partir du XIX ${ }^{\mathrm{e}}$ siècle (Kabatek et al., $2010: 257-258$ et 262ss respectivement). Dans le présent article, nous nous poserons cette question concernant le français : comment la textualité du français a-t-elle évolué entre le Moyen Âge et l'époque moderne?

Cette question est difficile à évaluer en raison des problèmes que pose la comparaison de textes d'époques différentes. Aussi bien les corpus de textes médiévaux et de leurs traductions modernes que les corpus réunissant des textes de ces deux époques censés se ressembler du point de vue de la typologie textuelle comportent des problèmes de comparabilité : les traductions conservent bien souvent des caractéristiques des textes de départ, et d'autre part, la typologie textuelle est très différente entre le français médiéval et le français moderne (Lavrentiev, 2007 : 153).

Pour remédier à ce problème et assurer une meilleure comparabilité de la textualité, cette étude se basera sur un corpus parallèle de deux traductions d'un même texte de l'Antiquité tardive, l'une provenant du XIV ${ }^{e}$, l'autre du XX siècle. Dans la mesure où la traduction du XX $\mathrm{X}^{\mathrm{e}}$ siècle se base sur le texte latin de l'édition moderne de Bieler (1957) et que celle du XIV ${ }^{e}$ siècle s'est également révélée suivre de très près le texte de cette édition (Hakulinen, 2007 : 25), les deux traductions donnent une représentation fiable d'un même texte à des époques différentes et permettent la comparaison de phénomènes textuels et linguistiques aux époques concernées. Ce type de corpus parallèle permet également de confirmer statistiquement des changements dans les fréquences relatives concernant divers phénomènes linguistiques à des époques différentes.

À travers ces deux textes seront donc mis en contraste deux états du français écrit, celui du XIV ${ }^{e}$ siècle où la langue écrite était jeune et les conventions de l'écriture n'étaient pas encore fixes, et le français de la première moitié $\mathrm{du} \mathrm{XX}^{\mathrm{e}}$ siècle, époque où elles sont à leur apogée et où l'influence d'internet ne s'est pas encore fait sentir. Le $\mathrm{XIV}^{\mathrm{e}}$ siècle est une période où la langue a subi de profonds changements, et c'est vers cette époque-là que l'on commence à produire pour la première fois dans l'histoire du français de plus en plus de textes d'utilité, non littéraires. Les traductions en langue 
vulgaire de textes latins de l'Antiquité ou médiévaux faites pour la nouvelle bourgeoisie des villes qui ne connaissait pas le latin ont un rôle non négligeable dans le processus de la formation textuelle de cette langue (Lusignan, 1987).

Les textes du corpus sont deux traductions françaises des deux époques concernées de la Consolatio Philosophiae de Boèce, texte latin datant de l'Antiquité tardive (début du VI ${ }^{\mathrm{e}}$ siècle), qui fut l'un des textes les plus lus et étudiés au Moyen Âge occidental ${ }^{1}$. Le texte médiéval du corpus, traduction anonyme qui figure sur un manuscrit de la fin du XIV ${ }^{\mathrm{e}}$ siècle ${ }^{2}$, reflète relativement bien la langue spontanée du XIV ${ }^{\mathrm{e}}$ siècle, notamment par son manque de structures et de vocabulaire latinisants (Hakulinen, 2007 : 20-21). De ce fait, il se prête bien à ce genre d'étude contrastive. La traduction moderne est de Bocognano (1937).

La textualité sera examinée à l'aide de la théorie des jonctions interpropositionnelles de Raible (1992, 2001). Les jonctions sont les manières de relier entre elles les propositions; elles contribuent au tissage textuel. Nous chercherons à déterminer de quelle manière le tissage textuel a évolué dans ce genre textuel particulier entre le XIV et le $\mathrm{XX}^{\mathrm{e}}$ siècle.

\section{Le tissage textuel et la proposition comme unité d'analyse}

Dans le modèle de Raible (1992, 2001), le tissage textuel est constitué par des jonctions, qui sont l'ensemble des moyens reliant les propositions entre elles. La nature d'une jonction entre deux propositions dépend de deux facteurs agissant simultanément : la présence d'un joncteur explicite éventuel (conjonction, connecteur ou préposition) et un moyen syntaxique, le degré de dépendance entre les propositions (juxtaposition, coordination, subordination). En fonction des liens textuels plus ou moins serrés qu'elles tissent, les jonctions interpropositionnelles peuvent être situées sur un continuum qui va de l'agrégation à l'intégration. Selon Raible (1992 : 303), dans l'agrégation extrême (exemple 1), le marquage linguistique de jonction est absent et les

\footnotetext{
${ }^{1}$ Il s'agit d'un ouvrage philosophique en cinq livres qui contient des monologues de Boèce et des dialogues entre Boèce et le personnage de la Philosophie. Il se compose d'une alternance de parties en prose et de parties en vers. Des centaines de manuscrits depuis le $\mathrm{IX}^{\mathrm{e}}$ jusqu'au $\mathrm{XV}^{\mathrm{e}}$ siècle en sont conservés, et en français seulement, il existe 13 traductions médiévales. Pour l'histoire de la Consolatio Philosophiae au Moyen Âge, voir Courcelle (1967) et Hakulinen (2007, chapitre 2 et la bibliographie).

${ }^{2}$ Le texte étudié provient du manuscrit Paris, BnF fr. 1728 (environ 1370-1380), qui est le plus ancien manuscrit contenant cette traduction anonyme dans son intégralité (la copie originale est perdue). Avec ses 64 manuscrits conservés, c'est aussi la traduction française la plus répandue de ce texte au Moyen Âge (Cropp, 1982-1983 : 263-264). La transcription du texte a été complétée, afin d'en combler des lacunes ou compléter des passages incompréhensibles, par deux manuscrits de contrôle, Vat. Reg. Lat. $1492\left(\mathrm{XV}^{\mathrm{e}}\right.$ s.) et Vat. Reg. Lat. 1508 (daté de 1362) ${ }^{2}$. La ponctuation du texte dans cette étude suit celle du manuscrit de Paris. Le manuscrit peut être consulté dans sa forme numérisée sur Gallica (http://gallica.bnf.fr). Le texte, qui s'intitule Le livre de Boece de Consolation, se trouve aux feuillets 223v-270v. Il a été copié et signé par Henri du Trévou.
} 
propositions sont entièrement indépendantes. Dans l'intégration extrême, à l'autre bout du continuum, c'est la fusion complète des propositions : une proposition est devenue un constituant d'une autre proposition (exemple 2: le SN la liberté de Rome est l'objet d'avoir souhaité) :

(1) On déclare que j'ai voulu sauver le sénat. Tu cherches comment? (Bocognano, 1937, 1P4, 20)

(2) pour m'accuser d'avoir souhaité la liberté de Rome (Bocognano, 1937, 1P4, 26)

Dans le modèle de Raible (1992, 2001), la cohésion phrastique est liée au degré de littératie dans la mesure où les jonctions d'un texte fortement caractérisé par la littératie ont tendance à être intégratives, serrées. Elles se caractérisent par la présence de techniques de condensation : subordination, formes verbales non finies et nominalisations de verbes. La langue orale ou un texte peu caractérisé par la littératie préfère en revanche les jonctions de type agrégatif, peu serrées : propositions autonomes, coordination ou juxtaposition. Une langue dispose d'une vaste palette de moyens d'expression lexicaux, grammaticaux et textuels, mais parmi ces moyens, l'utilisateur en choisit certains qui lui paraissent les plus opportuns, en grande partie en fonction de la tradition discursive dans laquelle il s'inscrit ( $c f$. Kabatek, Obris et Vincis, 2010 : 251). La langue étant un phénomène social, toutes les productions linguistiques s'inscrivent dans une certaine tradition : quel que soit le genre textuel en question, les auteurs qui tendent à l'adopter et à le produire se réfèrent à un modèle déjà existant et utilisent, consciemment ou inconsciemment, des éléments linguistiques qui lui sont typiques. Le degré d'intégration textuelle fait partie de ces éléments. Selon notre hypothèse, la traduction médiévale du corpus, produite avant l'existence d'une norme de l'écriture est potentiellement plus influencée par la langue orale et contient plus de propriétés agrégatives que la traduction moderne, plus caractérisée par la littératie et plus intégrative.

Le continuum entre oralité et littératie, ou le caractère oral ou écrit de la communication, a été discuté aussi par Koch et Oesterreicher (1985, 1994 : 590 et passim ; 2011 : 8, 16-18 et passim; voir aussi Koch 1995), qui font la distinction entre la langue de l'immédiat (Sprache der Nähe) et la langue de distance (Sprache der Distanz), l'immédiat étant caractéristique des textes oraux, et la distance des textes écrits ${ }^{3}$. Dans la même lignée d'idées, les travaux de Biber $(1995,2006)$ ont été parmi les premiers à montrer que ce même continuum constitue un facteur déterminant concernant le choix des divers éléments textuels.

\footnotetext{
${ }^{3}$ Selon ces auteurs, il ne s'agit pas du médium graphique ou phonique en tant que tel, mais de son sens figuratif ou " conceptionnel " : les caractéristiques qui seraient typiques des besoins communicatifs réalisés dans des situations de communication divergentes. Les textes oraux peuvent comporter des traits de distance (ex. les discours solennels) et les textes écrits des traits de l'immédiat (ex. le journal intime).
} 
Le modèle de Raible en tant que tel ne suffit pas à couvrir la diversité des cas de figure dans des textes réels. Pour affiner le modèle, nous avons proposé (Hakulinen et Larjavaara, 2018) d'ajouter la ponctuation comme troisième facteur contribuant au degré d'intégration d'un texte. La ponctuation, en permettant de segmenter un texte selon les choix de son auteur ou scripteur, contribue à son organisation syntaxique et textuelle ( $c f$. ., par exemple, Gautier, 2014 : 28). Dans la comparaison de la textualité de deux états de langue, le choix d'une ponctuation divergente peut indiquer un degré d'intégration différent.

L'unité d'analyse sera la proposition. C'est une unité syntaxique dont la forme canonique minimale se compose d'un SN sujet et d'un SV (Riegel, Pellat et Rioul, 2009 : 240 et 784-785). Dans ce travail, seules les propositions qui sont directement dominées par le niveau phrase seront prises en compte, étant donné que ce sont celles qui entretiennent les liens logiques principaux dans un texte. Les propositions compléments ou modifieurs de syntagme verbal ou nominal (c'est-à-dire les propositions complétives et relatives de la tradition grammaticale) sont conditionnées par des contraintes grammaticales et n'expriment pas (ou peu) de liens logiques ou circonstanciels de propositions (Matthiessen et Thompson, 2011 : 276-284) ${ }^{4}$. Par contre les séquences nominales sans SV, mais qui ont une fonction prédicative, sont des unités d'analyse, dans la mesure où elles s'interprètent comme des propositions de la même façon que les propositions syntaxiques 5 .

Les syntagmes verbaux contenant un gérondif - et fonctionnant en tant que circonstant - seront comptés comme des propositions avec un verbe non fini. La même chose vaut pour les participes présents, s'ils ne remplacent pas des propositions subordonnées relatives - dans ce dernier cas, ils sont modifieurs de SN. Les participes passés, en tant que formes adjectivales du verbe, ne seront pas retenus en tant que propositions.

L'analyse se base sur les propositions provenant des six parties en prose du premier livre ; elles sont au nombre de 371 en moyen français et 399 en français moderne, et elles ont été analysées selon la catégorisation de Raible ( $c f$. $\$ 4$ ci-dessous). Mais arrêtons-nous d'abord un instant sur la ponctuation, dont la comparaison entre deux époques lointaines demande une attention particulière.

\section{La ponctuation en tant qu'indication du degré d'intégration textuelle}

Malgré l'absence d'une norme de la ponctuation au Moyen Âge et du fait que celle-ci soit marquée par une grande variabilité dans les manuscrits ( $c f$. Marchello-Nizia, 1978 ; Llamas-Pombo, 2001 ; Lavrentiev, 2007, 2009, 2010), ce troisième

\footnotetext{
${ }^{4}$ Ce choix a une conséquence inévitable sur l'analyse : dans les deux versions du texte, on trouve des phrases longues qui se résument dans cette analyse à une seule proposition parce qu'elles se composent justement de SN et SV et de leurs compléments ou modifieurs.

${ }^{5}$ Ceci concerne également les séquences sans SV résultant d'une ellipse : Mais [ellipse] cestui qui a este nourriz de si parfondes sciences. (fr. 1728, 1P1,10).
} 
indicateur d'intégration textuelle à côté de la syntaxe et des joncteurs sera pris en compte dans cette étude, étant donné que la traduction médiévale contient également une ponctuation. Les marques de ponctuation sont employées systématiquement dans le passage étudié entier, mais elles reflètent probablement plus les choix personnels $\mathrm{du}$ scribe que les marques de la traduction moderne, qui sont placées en grande partie selon la ponctuation de l'éditeur moderne du texte latin - et en suivant les conventions modernes de l'écrit.

La ponctuation avérée dans un seul texte ne pouvant représenter de manière générale la situation au $\mathrm{XIV}^{\mathrm{e}}$ siècle, le marquage de la ponctuation du manuscrit de ce corpus a été considéré comme un témoin de texte authentique de son époque, et en tant que tel comme la réalisation d'un certain choix - celui du scribe - à propos de procédés contribuant à la textualité.

Pour qu'une comparaison de la ponctuation entre les deux états de langue soit possible, on aura besoin d'un tertium comparationis. Dans cette analyse, on adoptera la conception tripartite du degré de coupure entre les propositions : coupure "forte", " moyenne » ou "faible ", présente dans de nombreux traités sur la ponctuation depuis l'Antiquité ( $c f$. Lavrentiev, $2007: 157$; $2009: 36-37,85)^{6}$, auxquelles on associera les différentes marques de ponctuation relevées dans les deux traductions. Ainsi, dans la traduction moderne, le point, le point d'interrogation ou d'exclamation et l'alinéa correspondront à une coupure forte. Le point-virgule et le deux-points, dans la mesure où ces marques peuvent inclure en plus de la coupure, un lien logique de nature variable entre les propositions (Grevisse et Goosse, 2011 : \$129-130), seront considérés comme une coupure moyenne. La virgule marquera une coupure faible, tandis que l'absence de ponctuation représentera une absence de marquage de coupure. Il est clair que l'application mécanique d'un tel modèle ne peut être une solution parfaite pour attribuer dans chaque cas la vraie valeur d'un signe de ponctuation, mais nous l'appliquerons faute de mieux, tout en tenant compte que les résultats devront être observés avec précaution.

Pour la traduction médiévale du corpus, la valeur de la ponctuation a été déterminée en suivant Lavrentiev (2009 : 105, 126-128) : le degré (ou "force ") de coupure des marques de ponctuation se détermine par la variante - minuscule ou majuscule - de la lettre qui la suit, pas par le choix de la marque elle-même. Une majuscule ou une lettrine en couleur différente seule représente une ponctuation forte, une minuscule marque une ponctuation faible, quelle que soit la marque de ponctuation qui les précède, tandis qu'une "grande minuscule" (Lavrentiev, 2009: 127) représente une ponctuation moyenne. Le manuscrit $\mathrm{BnF}$ fr. 1728 présente les marquages suivants :

${ }^{6}$ Le Goffic (1993), par exemple, range les signes en trois groupes selon le degré de séparation syntaxique qu'ils imposent : les séparateurs forts (le point, le point d'interrogation, le point d'exclamation, le pointvirgule, les points de suspension et les deux points), les séparateurs faibles (la virgule) et les marques d'encadrement (les signes doubles, comme les guillemets ou les parenthèses par exemple). 
le " pied-de-mouche », toujours suivi d'une majuscule et le plus souvent précédé d'un point (ponctuation forte) ; le point (situé légèrement au-dessus de la ligne inférieure) suivi d'une majuscule (ponctuation forte) ou d'une minuscule (ponctuation faible) ; la barre oblique fine suivie d'une minuscule (ponctuation faible) et une fois d'une majuscule (ponctuation forte) ; la majuscule seule sans autre marque représente une ponctuation forte. Le passage étudié du manuscrit $\mathrm{BnF}$ fr.1728, écrit dans une écriture très calligraphique, ne comporte pas de "grandes minuscules ", et la ponctuation de force moyenne en est ainsi absente.

Le marquage de ponctuation situé aux frontières des propositions sera donc le troisième facteur contribuant au degré d'intégration du texte pris en compte dans cette étude. Il agit en corrélation avec les deux autres facteurs, la dépendance syntaxique entre les propositions et l'emploi des joncteurs.

\section{Entre agrégation et intégration : les catégories de jonctions}

Le Tableau 1 ci-dessous rend compte des occurrences de propositions dans les catégories de I à $\mathrm{V}$ du continuum de Raible ${ }^{7}$, augmentées par les informations sur la ponctuation et précisées selon les besoins de ce corpus particulier.

Les catégories I-III regroupent les propositions indépendantes (parataxe : elles ne dépendent syntaxiquement d'aucune autre proposition, et aucune proposition n'en dépend). La catégorie I renferme les propositions qui ne contiennent pas de joncteur, les propositions des catégories II et III sont introduites par un joncteur anaphorique (II) ou de coordination (III). Dans les catégories IV (verbe fini) et V (verbe non fini) sont rangées les propositions impliquées dans une subordination (hypotaxe), soit qu'elles dominent une subordonnée, soit qu'elles soient elles-mêmes subordonnées à une autre proposition. La catégorie V se divise en deux parties : I. Avec joncteur (propositions subordonnées réduites à l'infinitif, introduites par pour, afin de...), II. Sans joncteur (propositions subordonnées participiales ou gérondives : formes en -ant). Enfin, la ponctuation (forte, moyenne, faible ou absente) est prise en compte séparément dans chaque catégorie. Elle se détermine par rapport au contexte gauche de la proposition :

\footnotetext{
${ }^{7}$ Pour une présentation complète du modèle, $c f$. Raible (1992: 303) ainsi que Hakulinen et Larjavaara (2018: \$4.2). Le modèle de Raible comporte aussi les catégories VI et VII proches du pôle intégratif, mais leur intérêt reste théorique dans une analyse de cette sorte étant donné que les nominalisations et constituants verbaux présents dans les textes ne peuvent pas tous être mis en parallèle avec des constructions verbales virtuelles (Hakulinen et Larjavaara, 2018 : \$4.2).
} 


\begin{tabular}{|c|c|c|}
\hline Catégorie & $\mathrm{XIV}^{\mathrm{e}}$ s. (Paris, BnF, fr.1728) & $\mathrm{XX}^{\mathrm{e}}$ s. (Bocognano) \\
\hline (I) Pas de joncteur, proposi- & En tout : $91(24,40 \%)$ & En tout : $105(26,32 \%)$ \\
\hline $\begin{array}{l}\text { tion indépendante } \\
\text { a) Ponctuation FORTE }\end{array}$ & 56 & 63 \\
\hline b) Ponctuation MOYENNE & N & 19 \\
\hline c) Ponctuation FAIBLE & 19 & 23 \\
\hline d) Pas de ponctuation & 16 & - \\
\hline $\begin{array}{l}\text { (II) Joncteur anaphorique }{ }^{8} \text {, } \\
\text { proposition indépendante }\end{array}$ & En tout : $10(2,68 \%)$ & En tout : $5(1,25 \%)$ \\
\hline a) Ponctuation FORTE & 7 (dont 2 aussi dans IVa) & 4 \\
\hline b) Ponctuation MOYENNE & then & 1 \\
\hline c) Ponctuation FAIBLE & - & - \\
\hline d) Pas de ponctuation & 3 & + \\
\hline $\begin{array}{l}\text { (III) Joncteur de coordination, } \\
\text { proposition indépendante }\end{array}$ & En tout : $121(32,44 \%)$ & En tout : $95(23,81 \%)$ \\
\hline a) Ponctuation FORTE & 67 & 54 \\
\hline b) Ponctuation MOYENNE & 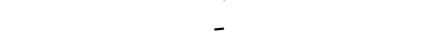 & 4 \\
\hline c) Ponctuation FAIBLE & 15 & 31 \\
\hline d) Pas de ponctuation & 39 & 6 \\
\hline $\begin{array}{l}\text { (IV) Joncteur de subordina- } \\
\text { tion, proposition subordonnée }\end{array}$ & En tout : $139(37,26 \%)$ & En tout : $152(38,09 \%)$ \\
\hline $\begin{array}{l}\text { à verbe fini } \\
\text { a) Ponctuation FORTE }\end{array}$ & 55 (dont 2 aussi dans IIa) & 45 \\
\hline b) Ponctuation MOYENNE & $x^{2}+2+2$ & 10 \\
\hline c) Ponctuation FAIBLE & 14 & 83 \\
\hline d) Pas de ponctuation & 70 & 14 \\
\hline (V) Joncteur de subordination, & En tout : $12(3,22 \%)$ & En tout : $42(10,53 \%)$ \\
\hline $\begin{array}{l}\text { proposition subordonnée à } \\
\text { verbe non fini }\end{array}$ & & \\
\hline $\begin{array}{l}\text { I. Avec joncteur : } \\
\text { a) Ponctuation FORTE }\end{array}$ & & \\
\hline $\begin{array}{l}\text { a) Ponctuation FORTE } \\
\text { b) Ponctuation MOYENNE }\end{array}$ & 2 & $\begin{array}{l}1 \\
2\end{array}$ \\
\hline c) Ponctuation FAIBLE & 1 & 7 \\
\hline d) Pas de ponctuation & 9 & 12 \\
\hline II. Sans joncteur : & & \\
\hline a) Ponctuation FORTE & - & 1 \\
\hline d) Ponctuation MOYENNE & - & 2 \\
\hline b) Ponctuation FAIBLE & - & 7 \\
\hline c) Pas de ponctuation & - & 10 \\
\hline TOTAL OCCURRENCES & $373^{9}(100 \%)$ & $399(100 \%)$ \\
\hline
\end{tabular}

Tableau 1. Les jonctions dans les deux traductions selon la catégorisation de Raible et la ponctuation.

\footnotetext{
${ }^{8}$ Les joncteurs anaphoriques renvoient explicitement à la proposition ou au texte qui précède (ou qui suit). Dans la traduction moderne nous avons voilà, voici (cataphorique) et voilà pourquoi, dans l'ancienne (et) pour ce, pour quoy et et de ce.

${ }^{9}$ Dans la traduction ancienne, deux occurrences appartiennent à la fois aux catégories IIa et IVa : proposition introduite par un joncteur anaphorique qui domine une subordonnée, ce qui augmente de 2 le nombre des propositions dans le tableau: Pour ce ne te merueilles de riens se tu vois que en la mer de ceste vie tempeste nous assaut $[\ldots](1 \mathrm{P} 3,11)$; Pour quoy combien que on mait ainsi fait / pleur ne ma pas encore si esbahi mon sens que ie ne me plaingne ou doie plaindre $(1 \mathrm{P} 4,28)$.
} 


\subsection{La syntaxe}

La catégorisation des propositions selon l'échelle de Raible fait apparaitre que l'organisation syntaxique de la traduction médiévale est effectivement plus parataxique, et donc plus agrégative, que celle de la traduction moderne : les catégories I, II et III représentent ensemble presque $60 \%$ des occurrences dans fr. 1728 contre env. $51 \%$ dans Bocognano. Cette différence de plus de 8 points est relativement marquée dans la mesure où il s'agit du même texte aux deux époques. Ce qui étonne cependant, c'est la distribution des occurrences dans les catégories : si certaines catégories soutiennent clairement ce constat, d'autres sembleraient, à première vue, aller à son encontre. La prise en compte des deux autres critères de la textualité (joncteurs, ponctuation) permet cependant d'expliquer ces divergences, comme nous le verrons plus bas.

L'indice syntaxique le plus clair d'une plus grande intégration dans la traduction moderne est assurément la nette différence dans les taux de la catégorie $\mathrm{V}$ : le taux des propositions avec un verbe non fini a augmenté de 3,22\% à 10,53\% (plus de 7 points de différence). La traduction en moyen français n'emploie jamais le verbe de la proposition dans une forme en -ant, bien que cette forme existe à cette époque (Buridant, 2000 : 254-255), alors qu'en français moderne, ces formes apparaissent 20 fois ; elles remplacent des propositions à verbe fini exprimant ici essentiellement la manière, ou des faits simultanés à une autre proposition (3). La traduction ancienne emploie à leur place des propositions coordonnées ou subordonnées à verbe fini (4) :

(3) en redressant la tête, elle arrivait à pénétrer dans le ciel même, échappant aux regards tournés vers elle. $\left(\mathrm{XX}^{\mathrm{e}} \mathrm{s} ., 1 \mathrm{P} 1,2\right)$

(4) et autre foiz la leuoit si haut que elle passoit le ciel sique iex domme ne la pouoient veoir (XIV s., 1P1, 2)

Les subordonnées réduites à l'infinitif sont aussi employées plus modérément dans la traduction en moyen français que dans la traduction moderne (12 occ. contre 22 occ.). En ce qui concerne l'autre catégorie intégrative, les propositions impliquées dans une subordination à verbe fini (catégorie IV), elle n'a pratiquement pas varié entre le moyen français et la période moderne (env. $38 \%$ dans les deux traductions).

Du côté du pôle agrégatif, la catégorie I de la traduction ancienne est légèrement moins importante que celle de la traduction moderne $(24,40 \%$ contre $26,32 \%$, soit presque 2 points de différence), ce qui est inattendu au vu des autres résultats. Par contre, les deux autres catégories agrégatives (II et III, regroupant les propositions indépendantes avec un joncteur anaphorique ou de coordination) sont chacune nettement plus importantes dans la traduction ancienne (pour la catégorie II, le taux y est double par rapport à la traduction moderne, soit 2,68 \% contre 1,25\% ; pour la catégorie III, la différence est de près de 9 points).

Pour mieux juger le niveau d'agrégation ou d'intégration des deux textes, ces chiffres seront mis en relation avec les données sur les joncteurs et la ponctuation, en commençant par les joncteurs. 


\subsection{Les joncteurs}

Si la dépendance syntaxique est un facteur grammatical du tissage textuel, les joncteurs tissent le texte surtout au niveau lexical. Les propositions introduites par un joncteur sont nettement plus fréquentes dans la traduction ancienne que dans la traduction moderne : dans la première, leur nombre total (seul ou combiné avec un autre joncteur) s'élève à presque $64 \%$, contre $53 \%$ dans la traduction moderne (11 points de différence). Du point de vue syntaxique, les propositions indépendantes avec ou sans joncteur (catégories I, II et III) forment une textualité agrégative. Dans la traduction ancienne, la séparation syntaxique des propositions est donc compensée par un emploi élevé des joncteurs, caractéristique qui tend vers l'intégration : les catégories II et III représentent 35,12\% des occurrences dans l'ancienne traduction, contre 25,06 $\%$ dans la traduction moderne (10 points de différence). La seule présence d'un joncteur sans dépendance phrastique contribue cependant à un tissage textuel au niveau du vocabulaire : le lien entre les propositions est formé par un moyen linéaire et non hiérarchique, ce qui est plus superficiel. De ce fait, le tissage demeure moins serré.

La tendance générale à favoriser la présence d'un joncteur dans la traduction ancienne fait que les propositions avec un joncteur anaphorique (catégorie II) y sont également plus nombreuses (10 occ. contre 5 occ. dans la traduction moderne). Or ce type de joncteurs, en créant à la fois un lien logique entre les propositions et en renvoyant à un antécédent plus ou moins précis dans le texte qui précède ou suit, contribue à un double tissage textuel et crée un lien plus serré par rapport aux autres joncteurs qui ne remplissent que la première de ces fonctions.

L'emplacement des joncteurs dans la proposition joue aussi un rôle dans le tissage textuel. Un joncteur placé à la frontière des propositions ne sert qu'à établir le lien entre les propositions : il tisse moins le texte qu'un joncteur placé plus loin dans la proposition et qui intègre toute la partie de la proposition qui le précède à la proposition précédente. Or dans la traduction moderne, on relève 13 occurrences (avec cependant, donc, en effet encore, enfin et pourtant) où le joncteur se trouve plus loin dans la proposition, alors que la traduction ancienne ne comporte que deux occurrences avec donques. Le tissage s'en trouve moins serré dans cette dernière.

Malgré une plus grande fréquence d'emploi des joncteurs, la traduction ancienne présente moins de variation dans leur choix : 37 joncteurs différents contre 44 dans la traduction moderne. L'abondance des joncteurs dans la traduction ancienne se manifeste aussi par le fait que certains joncteurs sont récurrents. En particulier le joncteur et, seul ou combiné avec un autre joncteur, y apparait 85 fois (35,3\% des propositions avec joncteur, contre 49 fois, soit $23,2 \%$ dans la traduction moderne), ce qui en fait presque un joncteur "passe-partout "; il sert de lien entre deux propositions mais n'indique que peu une relation logique. Ce type de joncteur contribue moins au tissage du texte qu'un joncteur qui indique une relation logique plus marquée. 
L'abondance du joncteur et ainsi que l'abondance générale des joncteurs dans les textes français médiévaux est connue ( $c f$. Buridant, 1980 : 16 ; Lavrentiev, 2007 : 153-154; 2009 : 82 ; Catach, 1996: 7 ${ }^{10}$. Le phénomène a été expliqué par le fait qu'en l'absence d'une ponctuation, caractéristique typique du langage moderne écrit dont la fonction est de resserrer le style sans l'intermédiaire des mots, les joncteurs sont là pour assurer la séparation et l'enchainement des segments du texte (Catach, 1996 : 7). Nos traductions contenant toutes les deux aussi bien des joncteurs qu'une ponctuation régulière, nous confronterons maintenant ces données avec celles sur l'emploi de la ponctuation.

\subsection{La ponctuation}

Dans une situation où les marqueurs principaux de la séparation et de l'enchainement des segments d'un texte sont censés être les joncteurs, comme il a été proposé pour les textes des états anciens du français (cf. ci-dessus), on s'attendrait à y trouver moins de marques de ponctuation que dans un texte moderne où les joncteurs sont censés jouer un rôle moindre. Le nombre total des propositions ponctuées est en effet bien plus élevé dans la traduction moderne que dans la traduction ancienne $(89,5 \%$ contre 63,5 \%, 26 points de différence). La fréquence des joncteurs supérieure dans la traduction ancienne vue ci-dessus (12 points de plus par rapport à la traduction moderne) ne compense donc pas le moindre emploi des signes de ponctuation : la traduction ancienne marque moins les jonctions par les joncteurs et la ponctuation que la traduction moderne. Ce « sous-marquage » du tissage textuel, où l'on indique moins la séparation et l'enchainement des segments du texte, doit être vu comme un signe d'agrégation dans la traduction ancienne.

La comparaison de la relation joncteur / ponctuation dans les deux traductions confirme cette tendance ( $c f$. le Tableau 2 ci-dessous) : les propositions sans joncteur et sans ponctuation représentent un taux de 11,6\% dans la traduction ancienne contre 3 $\%$ uniquement dans la traduction moderne, alors que le contraire, la cooccurrence d'un signe de ponctuation avec un joncteur, est moins fréquente dans la traduction ancienne que dans la traduction moderne (38,3\% contre 44,9\%: 6,6 points de différence). La préférence à marquer la jonction par un joncteur plutôt que par un signe de ponctuation dans la traduction ancienne se confirme également : le cas de figure +joncteur /-ponctuation représente $25,3 \%$ contre seulement $8 \%$ dans la traduction moderne et le contraire, +ponctuation/-joncteur, est bien plus fréquent dans la traduction moderne $(44,1 \%$ contre $24,8 \%)$.

${ }^{10}$ Ces auteurs parlent de conjonction ou de connecteur. 


\begin{tabular}{|c|c|c|}
\cline { 2 - 3 } \multicolumn{1}{c|}{} & $\mathrm{XIV}^{\mathrm{e}}$ s. (fr.1728) & $\mathrm{XX}^{\mathrm{e}}$ s. (Bocognano) \\
\hline - joncteur, - ponctuation & $11,6 \%(43)$ & $3 \%(12)$ \\
\hline + joncteur, - ponctuation & $25,3 \%(94)$ & $8 \%(32)$ \\
\hline - joncteur, + ponctuation & $24,8 \%(92)$ & $44,1 \%(176)$ \\
\hline + joncteur, + ponctuation & $38,3 \%(142)$ & $44,9 \%(179)$ \\
\hline TOTAL & $100 \%(371)$ & $100 \%(399)$ \\
\hline
\end{tabular}

Tableau 2 : La relation joncteur/ponctuation dans la traduction ancienne (fr.1728) et la traduction moderne (Bocognano).

Remarquons cependant que l'existence des règles de ponctuation augmente de toute évidence l'emploi de la ponctuation dans la traduction moderne, et du point de vue du tissage textuel, certaines de ces marques peuvent s'avérer redondantes avec les joncteurs. La redondance s'observe essentiellement dans les catégories III et IV : parmi les propositions indépendantes introduites par un joncteur et coordonnées à une autre proposition indépendante (III) ainsi que les propositions impliquées dans une subordination (IV), une marque de ponctuation faible (virgule) est nettement plus fréquente dans la traduction moderne, alors que la ponctuation est absente nettement plus souvent dans la traduction ancienne. La présence d'un joncteur ou l'implication dans une subordination, facteurs d'intégration textuelle, permettent l'absence d'une marque de ponctuation dans la traduction ancienne, alors que dans la traduction moderne, les règles de ponctuation imposent une virgule dans la plupart de ces cas-là (à l'exception généralement de la subordonnée qui suit une proposition dominante). La traduction ancienne comporte aussi plusieurs cas où une proposition subordonnée est séparée par une ponctuation forte à gauche et à droite, qui est un trait agrégatif (5):

(5) Auoie ie tel habit et tele chaiere quant ie cerchoie les secres de nature. Quant tu me declairoies les voies des estoilles a vne broche. Quant tu formoies noz meurs a la rayson de nostre vie a lexemple de lordre du ciel. (XIV s., 1P4,4)

Ce type de ponctuation, non normatif dans les conventions modernes, n'apparait pas dans la traduction moderne. Dans ce passage, cette dernière emploie une ponctuation faible devant chaque joncteur quand:

(6) Avais-je cette allure, avais-je ce visage, quand je sondais avec toi les mystères de la nature, quand avec ta baguette tu me traçais le mouvement des astres, quand tu réglais ma conduite et le plan de ma vie entière à l'image de l'ordre céleste? $\left(\mathrm{XX}^{\mathrm{e}}\right.$ s., $\left.1 \mathrm{P} 4,4\right)$

Les marques de ponctuation forte, moyenne ou faible construisent la textualité de manières différentes. Une ponctuation forte contribue à l'agrégation du texte. Or le taux total de la ponctuation forte est plus élevé dans la traduction ancienne que dans la traduction moderne $(50,4 \%$ contre $42,1 \%)$, et son emploi relatif dans chacune des catégories de I à $\mathrm{V}$ est également plus élevé. La ponctuation moyenne est absente dans la traduction ancienne, alors qu'elle est relativement fréquente dans la traduction moderne $(9,5 \%)$. Les marques de ponctuation moyenne augmentent l'intégration 
textuelle : au lieu de séparer les propositions, le deux-points peut signaler un lien sémantique entre deux unités linguistiques en annonçant «l'analyse, l'explication, la cause, la conséquence, la synthèse de ce qui précède " (Grevisse et Goosse, 2011: $\$ 130$ ), et le point-virgule est utilisé " entre phrases syntaxiquement autonomes mais associées du point de vue logique »(Grevisse et Goosse, 2011 : \$129). Quant à la ponctuation faible, qui ne sépare que faiblement les propositions, elle contribue plus à l'intégration qu'à l'agrégation textuelle. Comme nous venons de le voir, la ponctuation faible apparait nettement plus souvent dans la traduction moderne (36\% contre 13,1 $\%)$.

Tout autant que la ponctuation, l'absence d'une marque de ponctuation entre deux propositions, doit être considérée comme un facteur agissant sur l'intégration textuelle. Le taux total des propositions qui ne sont séparées par aucune ponctuation est de $36,7 \%$ dans la traduction ancienne contre $10,5 \%$ dans la traduction moderne. Toutefois, s'il est vrai qu'une coupure forte contribue à l'agrégation du texte et une coupure moyenne ou faible à son intégration, l'effet de l'absence de ponctuation sur la textualité dépend de son emplacement. Dans les catégories II, III, IV et V, où un autre facteur d'intégration (joncteur et/ou dépendance syntaxique) est déjà présent, l'absence de marque de ponctuation joue un rôle moindre de ce point de vue. Or la plupart des propositions sans ponctuation, soit $89 \%$, se trouvent dans ces catégories dans la traduction ancienne (121 occ. sans ponctuation sur 137 en tout) et toutes dans la traduction moderne (42 occ.).

La différence essentielle entre les traductions se trouve dans la catégorie I où faute de ponctuation aucun facteur d'intégration textuelle n'est présent : il s'agit d'une juxtaposition de propositions indépendantes sans joncteur ni ponctuation (7). La traduction moderne sépare les propositions par un point-virgule dans le même passage (8) :

(7) si estoit elle de si grant aage que nul homme ne si prenoit son grant nestoit pas dune mesure. (XIV e s., 1P1, 1)

(8) encore qu'elle fût si chargée d'ans qu'il était impossible de la croire de notre génération; sa taille ne pouvait se déterminer aisément. $\left(\mathrm{XX}^{\mathrm{e}}\right.$ s., $\left.1 \mathrm{P} 1,1\right)$

L'enchainement du type (7) étant proscrit par la norme de l'écrit moderne, la traduction moderne en est exempte, alors que la traduction ancienne en contient 16 occurrences $(4,3 \%)$. Les propositions sont présentées comme intimement liées, sans qu'une marque de ponctuation, ou un moyen syntaxique ou lexical, ne spécifie la relation sémantique qui s'instaure entre elles. La textualité s'en trouve peu serrée, caractéristique d'une textualité agrégative.

Dans le cas de la traduction médiévale, cette absence est évidemment due au fait que dans une situation où il n'existe pas de norme sur la ponctuation, chaque utilisateur de la langue est plus libre dans ses choix dans ce domaine. 


\section{En guise de conclusion}

Cette étude sur un corpus parallèle réunissant un texte du XIV ${ }^{e}$ et du XX $X^{e}$ siècle a examiné trois facteurs contribuant au degré d'intégration des textes : la dépendance syntaxique, l'emploi des joncteurs et la ponctuation. Pour chacun de ces facteurs, la textualité s'est complexifiée entre le Moyen Âge et l'époque moderne : la langue écrite jeune du XIV ${ }^{e}$ siècle présente effectivement plus de caractéristiques agrégatives que la langue de la première moitié du $\mathrm{XX}^{\mathrm{e}}$ siècle.

Nous avons vu cependant que les trois facteurs étudiés agissent de manière complexe sur le degré d'intégration des textes : le modèle de Raible, même complété par les données sur la ponctuation, n'est pas tel quel suffisant pour l'évaluer. Les facteurs d'intégration textuelle peuvent, selon les circonstances, tirer le texte vers des directions opposées sur l'axe agrégation - intégration. Une proposition syntaxiquement indépendante, par exemple, est une caractéristique agrégative, mais moins si elle est liée à une autre proposition par une ponctuation moyenne, et plus si elle en est séparée par une ponctuation forte, ou si la ponctuation manque totalement. La présence d'un joncteur ajoute au pouvoir d'intégration d'une jonction, mais plus encore s'il s'agit d'un joncteur anaphorique, ou si le joncteur est situé à l'intérieur et pas à l'exacte frontière de la proposition. D'autre part, un lien créé par un joncteur, moyen lexical et non syntaxique, est de nature linéaire et non hiérarchique, ce qui est moins intégratif qu'un lien créé par une dépendance syntaxique. De même, l'emploi fréquent et " mécanique " de certains joncteurs qui n'expriment pas clairement de lien logique (par exemple $e t$ dans le cas de la traduction ancienne) relèvent moins de l'intégration que l'emploi de joncteurs qui indiquent plus clairement une relation logique. Dans certains cas en revanche, une absence de joncteur peut être un signe d'une jonction très intégrative : c'est le cas des propositions subordonnées à verbe non fini. Quant à la ponctuation, une marque de coupure forte tire le texte vers l'agrégation, mais l'absence de marque de ponctuation, s'il n'y a pas d'autre marque de textualité entre deux propositions, peut le faire également. Le degré d'intégration d'un texte est donc le résultat d'une combinaison subtile de ces différents facteurs.

\section{RÉFÉRENCES BIBLIOGRAPHIQUES}

BIBER, Douglas (1995) : Dimensions of register variation: A cross-linguistic comparison. Cambridge, Cambridge University Press.

BIBER, Douglas (2006) : University Language: A Corpus-based Study of Spoken and Written Registers. Amsterdam / Philadelphia, John Benjamins.

BIELER, Ludwig (1957) : Anicii Manlii Severini Boethii Philosophiae consolatio. Turnhout, Brepols (Corpus Christianorum, Series Latina, 92). 
Bocognano, Aristide (1937) : Boèce, La Consolation de la Philosophie. Traduction nouvelle avec une introduction et des notes. Paris, Classiques Garnier.

BURIDANT, Claude (1980) : « Le strument et et ses rapports avec la ponctuation dans quelques textes médiévaux ", in Anne-Marie Dessaux-Berthonneau (éd.). Théories linguistiques et traditions grammaticales. Villeneuve-d'Asq, Presses Universitaires de Lille, 13-53.

BURIDANT, Claude (2000) : Grammaire nouvelle de l'ancien français. Paris, SEDES.

CATACH, Nina (1996 [1994]) : La ponctuation. Paris, Presses universitaires de France.

COURCELLE, Pierre (1967) : La consolation de Philosophie dans la tradition littéraire. Antécédents et postérité de Boèce. Paris, Études Augustiniennes.

Cropp, Glynnis M. (1982-83) : «Les manuscrits du 'Livre de Boece de Consolacion' ». Revue d'histoire des textes, 12-13, 263-352.

GAUTIER, Antoine (2014) : «Phrase et syntaxe : sur quelques aspects de l'intégration ». Langue française, 182, 27-41.

GREVISSE, Maurice \& André GOOSSE (2011) : Le bon usage. Grammaire française. Bruxelles, De Boeck Université [15édition].

HAKULINEN, Soili (2007) : La complémentation du verbe en moyen français et en français moderne. Etude diachronique sur la base d'un corpus parallele de traductions. Tampere, Tampere University Press (Acta Universitatis Tamperensis, 1274).

HAKUlinen, Soili \& Meri LARJAVAARA (2018) : «La littératie en voie de changement : respect du genre et jonctions interpropositionnelles dans des lettres à des professionnels de droit sur Internet ". Discours, 23. DOI : http://dx.doi.org/10.4000/discours.9818.

KABATEK, Johannes; Philipp OBRIST \& Valentina VINCIS (2010) : "Clause Linkage techniques as a symptom of discourse traditions: Methodological issues and evidence from Romance languages », in Heidrun Dorgeloh \& Anja Wanner (éds), Syntactic Variation and Genre. Berlin / New York, De Gruyter Mouton (Topics in English Linguistics, 70), 247-275.

$\mathrm{KOCH}$, Peter (1995) : "Subordination, intégration syntaxique et 'oralité' ", in Hanne Leth Andersen \& Gunver Skytte (éds), La subordination dans les langues romanes. Copenhague, Museum Tusculanum (Études Romanes, 34), 13-42.

KOCH, Peter \& Wulf OeSTERREICHER (1985) : «Sprache der Nähe - Sprache der Distanz: Mündlichkeit und Schriftlichkeit im Spannungsfeld von Sprachtheorie und Sprachgeschichte ». Romanistisches Jahrbuch, 36, 15-43.

KoCH, Peter \& Wulf OesTERREICHER (1994) : "Schriftlichkeit und Sprache », in Hartmut Günther \& Otto Ludwig (éds), Schrift und Schriftlichkeit - Writing and Its Use I, Berlin et New York, Walter de Gruyter, 587-604.

KOCH, Peter \& Wulf OesterReICHER (2011 [1990]) : Gesprochene Sprache in der Romania. Berlin et New York, De Gruyter (Romanistische Arbeitshefte, 31).

LAVRENTIEV, Alexei (2007) : «Pour une méthodologie d'étude de la ponctuation médiévale basée sur une approche typologique », in Bernard Combettes \& Christiane Marchello- 
Nizia (éds), Études sur le changement linguistique en français, communications du Colloque "Diachro 2 ». Nancy, Presses Universitaires de Nancy, 191-204.

LAVRENTIEV, Alexei (2009): Tendances de la ponctuation dans les manuscrits et incunables français en prose, du XIII au XVe siècle. Thèse de doctorat en Sciences du Langage sous la direction de Christiane Marchello-Nizia. Lyon, ENS LSH. URL : http://tel.archivesouvertes.fr/tel-00494914

LAVRENTIEV, Alexei (2010) : «La "phrase” en français médiéval : une réalité ou une reconstruction artificielle ?", in Frank Neveu et al, (éds) Congrès Mondial de Linguistique Française - CMLF 2010, Paris, Institut de Linguistique française, 277-289. DOI : https://doi.org/10.1051/cmlf/2010125.

LE GOFFIC, Pierre (1993) : Grammaire de la phrase française. Paris, Hachette.

LLAMAS-POMBO, Elena (2001) : «La ponctuation du vers dans un manuscrit du XIV siècle ». Liaisons HESO/AIROE, 32-33, 151-171.

LUSIGNAN, Serge (1987) : Parler vulgairement. Les intellectuels et la langue française aux XIII et XIV siècles. Paris, Vrin.

MARCHELlO-NIZIA, Christiane (1978) : «Ponctuation et "unités de lecture" dans les manuscrits médiévaux ou : je ponctue, tu lis, il théorise ». Langue française, 40, 32-44.

MATTHIESSEN, Christian \& Sandra A. THOMPSON (2011 [1988]) : "The structure of discourse and subordination ", in John Haiman \& Sandra A. Thompson (éds), Clause Combining in Grammar and Discourse. Amsterdam / Philadelphia, John Benjamins, 275-329.

RAIBLE, Wolfgang (1992) : Junktion: Eine Dimension des Sprache und ihre Realisierungsformen zwischen Aggregation und Integration. Heidelberg, Winter (Sitzungsberichte der Heidelberger Akademie der Wissenschaften, Berichtheft, 2).

RAIBLE, Wolfgang (2001) : "Linking clauses », in Martin Haspelmath, Ekkehard König, Wulf Oesterreicher \& Wolfgang Raible (éds), Language Typology and Language Universals, Volume 1 : An International Handbook. Berlin / New York, de Gruyter, 590-617.

RIEGEL, Martin; Jean-Christophe PELLAT \& René Rioul (2009 [1994]) : Grammaire méthodique du français. Paris, PUF. 